\title{
Improving itaconic acid production through genetic engineering of an industrial Aspergillus terreus strain
}

Xuenian Huang ${ }^{1,3}$, Xuefeng Lu', Yueming $\mathrm{Li}^{2}$, Xia $\mathrm{Li}^{2}$ and Jian-Jun $\mathrm{Li}^{1{ }^{*}}$

\begin{abstract}
Background: Itaconic acid, which has been declared to be one of the most promising and flexible building blocks, is currently used as monomer or co-monomer in the polymer industry, and produced commercially by Aspergillus terreus. However, the production level of itaconic acid hasn't been improved in the past 40 years, and mutagenesis is still the main strategy to improve itaconate productivity. The genetic engineering approach hasn't been applied in industrial A. terreus strains to increase itaconic acid production.
\end{abstract}

Results: In this study, the genes closely related to itaconic acid production, including cadA, mfSA, mttA, ATEG_09969, gpdA, ATEG_01954, acoA, mt-pfkA and citA, were identified and overexpressed in an industrial A. terreus strain respectively. Overexpression of the genes cadA (cis-aconitate decarboxylase) and $m f s A$ (Major Facilitator Superfamily Transporter) enhanced the itaconate production level by $9.4 \%$ and $5.1 \%$ in shake flasks respectively. Overexpression of other genes showed varied effects on itaconate production. The titers of other organic acids were affected by the introduced genes to different extent.

Conclusions: Itaconic acid production could be improved through genetic engineering of the industrially used $A$. terreus strain. We have identified some important genes such as cadA and $m f s A$, whose overexpression led to the increased itaconate productivity, and successfully developed a strategy to establish a highly efficient microbial cell factory for itaconate protuction. Our results will provide a guide for further enhancement of the itaconic acid production level through genetic engineering in future.

Keywords: Itaconic acid, Aspergillus terreus, Genetic engineering, Itaconate titer

\section{Background}

Itaconic acid is on the DOE (Department of Energy) top 12 list of the biotechnologically produced key building blocks in chemicals production, and is a valuable monomer or co-monomer in polymers manufacturing [1,2]. In future, it can even replace methacylic acid which is presently produced by the petrochemical industry [3]. Since the 1960s it has been produced by fermentation with Aspergillus terreus. Although several other natural itaconate producers such as Ustilago maydis and Pseudozyma antartica have been discovered, they couldn't compete

\footnotetext{
*Correspondence: lijj@qibebt.ac.cn

'Key Laboratory of Biofuels, Shandong Provincial Key Laboratory of Energy Genetics, Qingdao Institute of Bioenergy and Bioprocess Technology, Chinese Academy of Sciences, No. 189 Songling Road, Qingdao 266101, China

Full list of author information is available at the end of the article
}

with $A$. terreus based on the production level of itaconic acid [1].

As far as the biosynthesis of itaconic acid is concerned, there has been a lot of debate for a long time, and it still hasn't been fully established and understood [1,4-6]. It is generally accepted that the biosynthetic pathway occurs in two compartments: the cytosol and the mitochondria (Figure 1) $[1,4,5,7]$. The supposed pathway starts with glycolysis in the cytosol. Glucose is metabolized into pyruvate through the EMP pathway (Embden-Meyerhof-Parnas). Pyruvate is then either transported to the mitochondria and converted into acetyl-CoA, or carboxylated into oxaloacetate in the cytosol. Oxaloacetate is then transformed into malate, which is transported into the mitochondria by the malate/citrate antiporters. In the mitochondria, acetyl-CoA and oxaloacetate were condensed into citric acid by citrate synthase (CS). Citric acidis converted into 


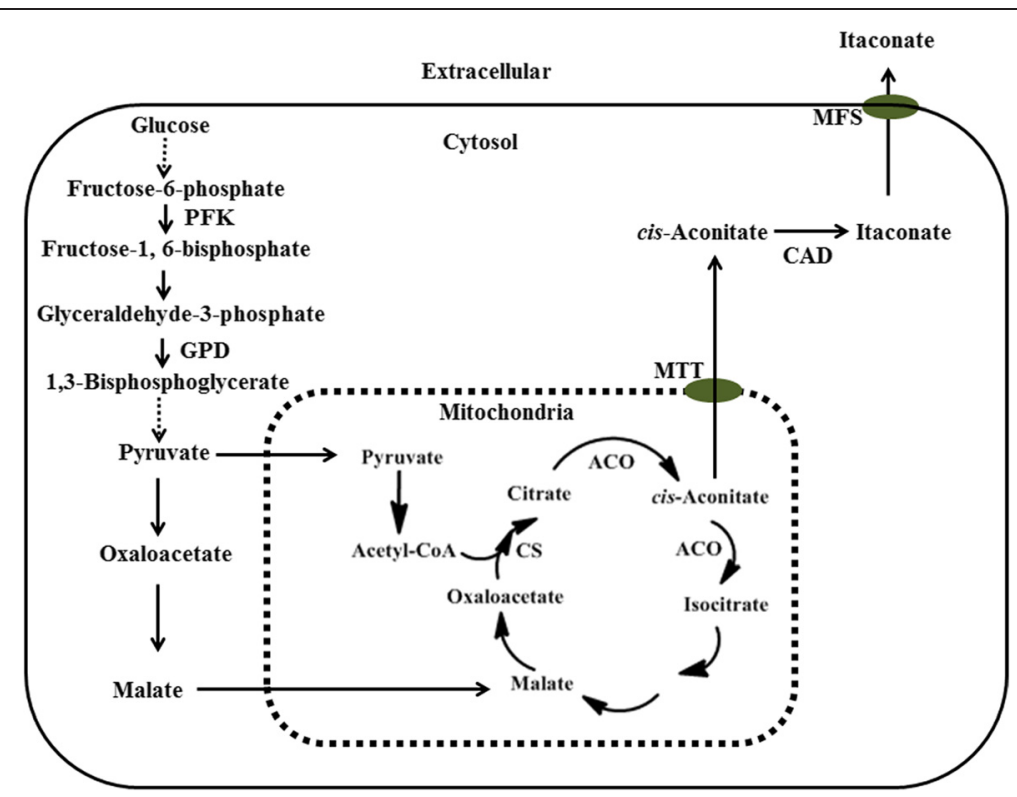

Figure 1 Biosynthetic pathway of itaconic acid in Aspergillus terreus [1,5-7]. The enzymes which were investigated in this study are shown in the figure. Abbreviations: PFK:6-phosphofructo-1-kinase; GPD: glyceraldehydes-3-phosphate dehydrogenase; ACO: aconitase; CS: citrate synthase; MTT: mitochondrial tricarboxylic transporter; CAD: cis-aconitate decarboxylase; MFS: major facilitator superfamily.

cis-aconitate by aconitase (ACO), which is transported back into the cytosol with the help of mitochondrial tricarboxylic transporter(s) (MTT) and then decarboxylated into itaconate by cis-aconitate decarboxylase (CAD). Finally, itaconic acid is exported out of the cell likely via the di-carboxylic acid carrier.

Recently, Li et al. identified the gene cluster of the itaconic acid biosynthesis in A. terreus through a clonebased transcriptomics approach [5], comprising $m t t A$ (ATEG_09970) encoding the putative MTT which is suggested to function in transporting tricarboxylate such as citrate, cis-aconitate and isocitrate from the mitochondria to the cytosol and/or vice versa, $\operatorname{cadA}$ (ATEG_09971) encoding CAD which has been proved to be essential in the itaconic acid biosynthesis [8,9], $m f_{s} A$ (ATEG_09972) encoding the putative MFS (Major Facilitator Superfamily) transporter which is a diverse family of transporter proteins, transporting compounds ranging from sugars to organic acids, including dicarboxylic acids such as itaconic acid, and ATEG_09969 encoding the putative regulator protein which contains a zinc finger motif characteristic of the eukaryotic transcription factors. This is a great scientific breakthrough in the field of itaconic acid. However, the actual regulation of the itaconate biosynthesis has not yet been fully understood.

Despite 60 years of studies on itaconate production, the current titers are almost same as those of 40 years ago [10]. So far, mutagenesis and process optimization are still the main approaches to improve strains for higher itaconic acid production [1,11-13]. However, there is a need for further cutting down the cost of itaconate production, which could be realized by increasing the efficiency of itaconic acid production, achieving higher titers, and utilizing the cheap and sustainable substrates [1]. Genetic engineering can be applied to come to a breakthrough in this microbial production process.

There have been some examples of improving the production level of itaconic acid through genetic engineering. For instance, Tevžet al. reported that itaconic acid accumulation in $A$. terreus could be enhanced by deregulating the glycolytic flux that was achieved by introducing a gene encoding a highly active, citrate inhibition resistant 6-phosphofructo-1-kinase mutant (mt-PFK1) [14]; Expression of the Vitreoscilla hemoglobin gene in A. terreus and a fungal haemoglobin domain in itaconate-producing $A$. niger also improved the itaconic acid titer due to increased oxygen uptake [15,16]; Expression of $m t t A, m f_{s} A$ and mt-pfkA (encoding the 6-phosphofructo-1-kinase mutant) in itaconate-producing $A$. niger increased itaconate productivity [16,17]; Expression of the itaconic acid cluster consisting of $c a d A, m t t A$ and $m f s A$ in $A$. niger led to a twenty-fold increase in itaconate yield compared to a strain expressing only $\operatorname{cad} A$ [18]. However, the $A$. terreus and $A$. niger strains used in the literature are nonindustrial ones [14-19].

Moreover, some genes which are possibly related to itaconic acid production have been discovered. For example, the transcription levels of the $g p d A$ gene encoding glyceraldehydes-3-phosphate dehydrogenase (GPD) (ATEG_09817) and ATEG_01954 (a predicted protein) 
dramatically increased 15.8 -and 14.5-fold respectively under the optimal itaconate production conditions [5], and that of the acoA gene encoding aconitase (ACO) also increased under itaconate production conditions [7]. Furthermore, considering that citric acid is a key precursor of the itaconic acid biosynthesis, citrate synthase (CS), which is encoded by citA and catalyses an irreversible reaction, may play an important role in improving itaconic acid production.

The genetic engineering approach has been extensively used for filamentous fungi to improve the production level, and produce the novel tailored compounds or direct the synthesis of the desired compounds [20,21], but it has only been utilized in a few examples to improve itaconic acid production by non-industrial $A$. terreus and $A$. niger strains [14-19]. In the current study, the genes closely related to the itaconic acid biosynthesis were identified and used to improve itaconate production through genetic engineering of industrially used $A$. terreus strains.

\section{Results and discussion}

Gene cloning, construction of the expression cassettes, transformation and analysis

Since it has been realized that right compartmentalization of the overexpressed proteins is important for itaconate production [22], all candidate genes include their native signal peptides when they were cloned.

Mutant mt-pfkA from A. niger Co827 was made as reported [23]. The genes from $A$. terreus were amplified by PCR using the cNDA of $A$. terreus LYT10 as the template and the respective primer pairs. The coding sequences (CDSs) of most cloned genes showed high identities to the annotated ones in the genome sequence of $A$. terreus $\mathrm{NIH}$ 2624, and the sequence identities between them are $>96 \%$ and $>98 \%$ for CAD, MTT, CS and ATEG_01954 at the gene and protein levels respectively. However, the sequence identities between cloned $m f s A$, ATEG_09969, gpdA, acoA and the annotated ones are $80.5 \%$ to $93.2 \%$ and $81.5 \%$ to $92.8 \%$ at the gene and protein levels respectively. Since the functions of most annotated genes in the genome sequence of $A$. terreus NIH2624 were predicted only and have not been characterized yet, the big difference in CDSs between the cloned and annotated genes is possibly caused by designation of introns or/and exons or/and translation start sites or/and stop codons. The CDSs of $m f s A$, ATEG_09969, gpdA and acoA were deposited in GenBank with the accession numbers KF305087, KF305088, KC213825 and KF268034, respectively.

Most candidate genes were inserted into the vector pAN52-4 at the restriction sites of $\mathrm{NcoI}$ and $\mathrm{BamHI}$ or HindIII respectively [24]. The obtained constructs were co-transformed with pAN7-1 harbouring the hygromycin resistance gene into $A$. terreus LYT10. The integrated target genes were confirmed by genomic PCR (Additional file 1: Figure S1). ATEG_01954 was cloned into the vector pG3H at the restrictions sites of PciI and BglII [25], and the generated plasmid was linearized and transformed into A. terreus LYT10. The transformants were selected on PDA-SH plates. The copy numbers of the introduced genes were determined by Southern blot. Since it is still controversial about the relationship between the copy numbers and translation/transcription [26-28], the copy numbers were only determined for the chosen cadAand $m f s A$-transformants (Additional file 2: Figure S2). Multi-copy genes were detected in most cadA- and $m f s A$-transformants, for example, 2-, 4-, 3-, 1-, and 2-copy of the cadA gene were integrated in the transformants cadA-2, $-5,-18,-21$ and -22 respectively (Additional file 2: Figure S2A), whereas 1-, 4-, 4-, 8-, and 2-copy of the $m f s A$ gene were present in transformants $m f s A-2$, $-10,-17,-12$ and -24 respectively (Additional file 2: Figure S2B). It is worth pointing out that the endogenous cadA band was not observed in the cadA-2 transformant (Lane 2, Additional file 2: Figure S2A), indicating that homologous recombination occurred for it. Thus, one band should be from homologous recombination, and the other one is from random integration (Lane 2, Additional file 2: Figure S2A).Our results also indicated that there is no obvious relationship between the copy numbers of the introduced genes and the itaconate titer. Similar results were also observed by Tevž et al. when mt-pfkA was overexpressed in A. terreus [14].

The transformants which can grow healthily on PDA plates were tested for itaconic acid production by cultivation in shake flasks for $76 \mathrm{~h}$ at $37^{\circ} \mathrm{C}$. Triplicate experiments were performed for each transformant, and were repeated for the better transformants at least once.

\section{Effects of respective overexpression of $\operatorname{cad} A, m f s A, m t t A$} and ATEG_09969 in A. terreus on itaconic acid production Remarkably, twenty-three out of twenty-four cadAtransformants produced higher itaconic acid titers than WT (Additional file 3: Figure S3A) (Figure 2), among which the best one - cadA-21 produced itaconic acid up to $88.1 \mathrm{~g} / \mathrm{L}, 9.4 \%$ higher than the parental strain, indicating that overexpression of cadA in A. terreus is beneficial for itaconate production (T-test, $\rho=0.000$ ) (Figure 2). These results demonstrated that introduction of the $\operatorname{cadA}$ gene contributed to itaconate production, and CAD was functional when overexpressed in A. terreus LYT10. Var-

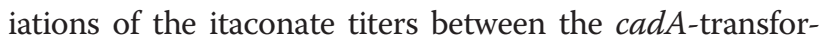
mants were observed (Additional file 3: Figure S3A) (Figure 2), which might be possibly due to heterologous recombination in $A$. terreus and/or growth effects. In fact, this is the first time that $\operatorname{cadA}$ was overexpressed in $A$. terreus, particularly an industrial $A$. terreus strain. Our results were consistent with the observation that the transcription level of cadA increased under the itaconic acid 


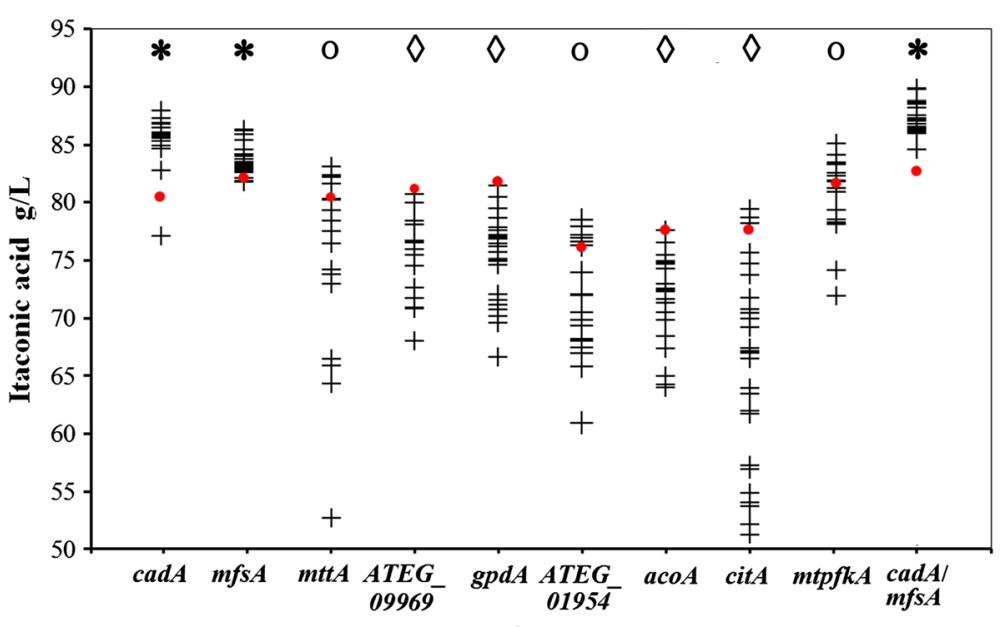

Transformants

Figure $\mathbf{2}$ Itaconic acid production by the transformants of different genes. The transformants of the different genes were screened for itaconate production. Each transformant was grown in $50 \mathrm{ml} \mathrm{IPM}$ on a rotary shaker at $37^{\circ} \mathrm{C}$ for $76 \mathrm{hr}$ in three individual flaks. The titers of itaconic acid produced were determined by HPLC. WT was represented by the solid red circle. *: statistically significant $(\rho<0.05$, beneficial for itaconate production). $\bullet$ : statistically significant ( $\rho<0.05$, adverse for itaconate production). o: statistically non-significant ( $\rho>0.05$, no impact on itaconate production).

producing conditions and that of $\operatorname{cad} A$ was approximately five-fold higher in the high-producing strain than that in the wild-type strain $[5,8]$, and also confirmed that CAD is a key enzyme involved in the itaconic acid biosynthesis and the CAD-catalyzed reaction might be a rate-limiting step for itaconate production. Because of its key role in the itaconic acid biosynthesis, E. coli, Y. lipolytica and $A$. niger have been engineered to produce itaconic acid by introducing the cadA gene [5,18,22,29-31]. However, the highest titer only reached $6 \mathrm{~g} / \mathrm{L}$ [29], which is far below the current one of $A$. terreus.

All twenty-six $m f s A$-transformants showed slightly higher itaconic acid productivity than the parental strain (Additional file 3: Figure S3B) (Figure 2), in which the itaconate titer of the best one - $m f s A-16$ is $5.1 \%$ higher than that of WT, demonstrating that overexpression of $m f_{s} A$ in $A$. terreus is beneficial for itaconate production (T-test, $\rho=0.045$ ) (Figure 2). The results were in good agreement with the reported ones where all 24 transformants produced higher itaconate titers than the parental strain while $m f s A$ was introduced into itaconate-producing $A$. niger, and demonstrated that MFS might act as an itaconate exporter and play important roles in itaconic acid production $[5,16]$. Overexpression of $m f s A$ could lead to faster transporting itaconic acid produced in the cytosol out of the cell, driving the metabolic flux toward itaconate biosynthesis, and enhancing the itaconate titer, though the improvement is not that high as overexpression of $c a d A$.

The putative MTT (ATEG_09970), which might act as a citric acid and/or cis-aconitate carrier, was overexpressed in A. terreus LYT10. Most $m t t A$-transformants showed slightly lower itaconic acid titers than WT (Figure 2), demonstrating that introduction of $m t t A$ into $A$. terreus is not beneficial for itaconate production (T-test, $\rho=0.24$ ). Our results were consistent with the ones of Li et al:: only one transformant exhibited higher itaconic acid production level among eight $m t t A$-transformants of itaconic acid producing $A$. niger, and they observed that the $m t t A$ transformants showed the increased accumulation level of citric acid [16]. However, van der Straat et al. found that expression of $m t t A$ resulted in a twenty-fold increase in the secretion of itaconic acid in A. niger [18]. It is still not clear why different results were obtained for the $m t t A$ transformants of $A$. terreus and $A$. niger. The fact that the equilibrium of the ACO-catalyzed reaction lies at the side of citrate with more than $90 \%$ and almost no citrate could be detected in cultures of itaconate producing $A$. terreus implies that very quick removal of cis-aconitate through MTT (or the cis-aconitate carriers) and/or CAD is very important for itaconic acid production [1]. A lot of studies on metabolite transport across the mitochondrial membrane have been carried out, for example, a novel transporter for dicarboxylates and tricarboxylates in the plant mitochondria has been identified [32], and a mitochondrial citrate transport protein from the yeast S. cerevisiae has been functionally characterized [33]. However, the mechanism of the mitochondrion carrier proteins related to organic acid transport in fungi is still not very clear. Accordingly, the function of MTT (ATEG_09970) awaits further research. Blumhoff et al. tried to address this issue in $A$. niger through co-overexpressing cadA and acoA in the cytosol and mitochondria [22].

When ATEG_09969 was introduced into A. terreus LYT10, most transformats produced slightly lower titers 
than WT (Figure 2), indicating that overexpression of ATEG_09969 into A. terreus is adverse for itaconate production (T-test, $\rho=0.014$ ). Based on the speculation that ATEG_09969 might regulate itaconic acid production at the transcriptional level, overexpression of it could contribute to the higher itaconate production level [5]. However, our results didn't support this. Actually, ATEG_09969 is also directly linked to the lovastatin gene cluster and may play parts in the lovastatin biosynthesis, since it has been reported that lovastatin and its intermediates were not detected while ATEG_09969 (equivalent to $l o v H$ or ORF13 in the lovastatin gene cluster) was rendered inactive via mutation [34]. In addition, Lai et al. found that the production levels of itaconic acid and lovastatin are inversely related [35]. Further study is required to clarify the function of ATEG_09969 in the itaconic acid biosynthesis.

\section{Effects of respective overexpression of $g p d A$,} ATEG_01954, acoA, citA and mt-pfkA on the itaconic acid titers

When gpdA, ATEG_01954, acoA and citA were overexpressed in $A$. terreus LYT10 respectively, most transformants showed lower itaconate production level than the parental $A$. terreus strain (Figure 2). The effects of overexpression of gpdA, ATEG_01954 and acoA on the itaconate titers were not expected based on the transcription data [5,7], and the screening results were discussed as follows: (1) Li et al. also observed that a true gpdA transformant (containing 4 extra gene copies) showed lower itaconate production level than WT, but they didn't explain why [19]. It has been realized that the requirement of an overall redox balance within the cell is a key factor determining the overall yields and productivities of a given product $[36,37]$. Since GPD is an $\mathrm{NAD}^{+}$dependent enzyme, overexpression of GPD in A. terreus might result in the imbalance of the redox cofactors, which would possibly affect itaconate production. (2) ATEG_01954 is a predicted small protein of 189 amino acids, whose function is unknown, so further research is required to elucidate its performance, its function in particular. (3) Given the fact that overexpression of $a c o A$ might not only contribute to the cis-aconitate pool, but also possibly accelerated conversion of cis-aconitate into isocitrate, it might be understandable that introduction of extra copies of aco $A$ showed no obvious impact on the itaconate titer. However, based on the results of the cadA-transformants, overexpression of $c a d A$ could drive carbon flux towards itaconic acid biosynthesis, and it is possible to improve itaconic acid production through co-expression of $a c o A$ and $\operatorname{cadA}$. Blumhoffet al. found that co-overexpression of $a c o A$ and $\operatorname{cad} A$ in the mitochondria doubled itaconate productivity compared with strains overexpressing both enzymes in the cytosol [22]. In addition, it has been reported that overexpression of ACO in Yarrowia lipolytica did not result in a shift of the CA (citric acid) /ICA (isocitric acid) product pattern into the direction of ICA [38]. (4) The results of $c i t A$-transformants implied that the reaction catalysed by CS might not be rate-limiting or CS contributes little to the flux control in the itaconic acid biosynthesis. Likewise, overexpression CS in A. niger didn't increase the citric acid production level [39].

It has been assumed that the strong anaplerotic reactions that replenish the pool of the TCA cycle intermediates would enhance the synthesis and excretion rate of itaconic acid, which could be realized by a highly active, citrate inhibition-resistant shorter PFK1 fragment (mt-pfkA) [14]. Only five out of nineteen mt-pfkA-overexpressing transformants produced slightly higher itaconic acid titers than WT (Figure 2). Statistically (T-test, $\rho=$ 0.448 ), overexpression of $\mathrm{mt}-p f k A$ in $A$. terreus is not beneficial for itaconate production (Figure 2). So, the production level of itaconic acid caused by overexpression of mt-pfk $A$ was not significantly enhanced as reported [14]. The different performance of the mt-pfkA transformants could possibly arise from the fact that the different hosts were used, a hyper itaconic acid producer $(\sim 80 \mathrm{~g} / \mathrm{L})$ was utilized in our case, whereas a poor one $(13.5 \mathrm{~g} / \mathrm{L})$ was used by Tevž et al. [14].

\section{Effects of co-overexpression of cadA and $m f s A$ in A. terreus on itaconic acid production}

Inspired by the performance of the $c a d A$ - and $m f s A$-transformants, $c a d A$ and $m f_{s} A$ were co-expressed in $A$. terreus LYT10 to see if there are any additive beneficial effects on itaconic acid production. All 20 co-transformants of cadA and $m f s A$ produced higher itaconate titers than the parental strain, among which the titer of the best one $c a d A$ / $m f s A-19$ is $8.7 \%$ higher than that of WT (Additional file 4: Figure S4) (Figure 2), demonstrating that co-expression of $c a d A$ and $m f_{s} A$ in $A$. terreus contributes to itaconate production (T-test, $\rho=0.000$ ). Unexpectedly, the cotransformants didn't exhibit the obvious additive beneficial effects. It appears that the co-transformants are slightly better than the cadA-transformants, and much better than the $m f s A$-transformants (Figure 2), further proving the importance of $\operatorname{cad} A$ initaconate production. These results might imply that the unknown bottlenecks or factors could limit the performance of the co-transformants and need to be further identified.

\section{Itaconic acid production by the transformant cadA-21 at the pilot scale}

The best itaconic acid producer $\operatorname{cadA-21}$ was tested at the demonstration scale ( $35 \mathrm{~m}^{3}$ fermentor). A two-stage process including the vegetative and production phase was used [40]. The growth of the transformant $\operatorname{cad} A-21$ was nearly not affected (data not shown). Itaconic acid 
produced by cadA-21 was analysed and quantified by HPLC (High Performance Liquid Chromatography). Based on the HPLC results, the titer of itaconic acid in the final fermentation culture of cadA-21 was similar to the parental strain, around $78.5 \pm 2.2 \mathrm{~g} / \mathrm{L}$ (Additional file 5: Figure S5) (Additional file 6: Figure S6A). However, it consumed glucose slightly more quickly (Additional file 6: Figure S6B). Thereby the production stage was shortened for around $3 \mathrm{hr}$, indicating that over-expression of cadA in A. terreus didn't have negative effect on the metabolic flux towards itaconate biosynthesis, but slightly accelerated that process.

\section{The effects of gene overexpression on extracellular organic acids rather than itaconic acid}

The concentrations of extracellular organic acids produced by some strains were determined (Table 1 ). The citrate concentrations were estimated to be below $0.01 \mathrm{mM}$, while pyruvate, lactate and oxalate could not be detected in all samples.

All selected strains showed lower $\alpha$-ketoglutarate titers in comparison with the parental strain. The cis-aconitate titers were improved by overexpression of citA, $m f_{s} A$, $m t t A$ and $m t-p f k A$, whereas it was greatly reduced by introduction of $g p d A$. The malate titers were significantly decreased by overexpression of citA and acoA. Overexpression of $m t t A$ led to significantly enhanced succinate titer, while most transformants showed lower succinate production level than WT, strain citA-7 in particular. The fumarate titers of most strains were not affected too much, except those of mttA-16, ATEG_09969-14 and $a \operatorname{coA}-20$.

Our results demonstrated that accumulation of cis-aconitic acid is important for itaconate production and the titers of organic acids were affected by the introduced genes to different extent. The observation that the itaconate concentration (around $600 \mathrm{mM}$ ) is significantly higher than the citrate one (below $0.01 \mathrm{mM}$ ) reflected the special itaconate accumulation mechanism in A. terreus.

\section{The regulation of the itaconate biosynthesis in $A$. terreus}

Based on the studies towards citric acid production by $A$. niger, Torres et al. used the biochemical systems theory coupled with constrained linear optimization to show that at least seven enzymes need to be co-expressed to achieve a significant increase in flux towards the citrate biosynthesis [41], and predicted that adjusting the step that removes the desired product constituents the most promising metabolic engineering strategy, implying that manipulation of the transporters involved in citrate uptake and export would be a desirable strategy for increasing the rate of citrate production.

Since the biosynthesis of itaconic acid in A. terreus is very similar to that of citric acid in $A$. niger in some aspects, the same theory might be applicable to itaconate production by $A$. terreus and help to explain some above results. Respective overexpression of the central carbon metabolism genes such as mt-pfkA, gpdA, citA and acoA didn't increase itaconate productivity, while over-expression of $m f s A$, the potential itaconate exporter, led to the enhanced itaconate production level. However, overexpression of $\operatorname{cadA}$, resulting in the enhanced itaconate titer, couldn't be explained by this theory. Thus, a different theory may be required for itaconic acid production.

\section{Conclusions}

In conclusion, the genes which are closely related to itaconate production were identified and overexpressed in an industrially used $A$. terreus strain, including $\operatorname{cad} A$, $m f s A, m t t A$, ATEG_09969, gpdA, ATEG_01954, acoA, cit $A$ and mt-pfkA. Overexpression of $c a d A$ and $m f s A$ enhanced the itaconate production level, and introduction of

Table 1 Extracellular organic acids $(\mathrm{mM})$ produced by different strains ${ }^{\mathrm{a}}$

\begin{tabular}{|c|c|c|c|c|c|c|}
\hline Strain & Itaconic acid & cis-aconitic acid & Malic acid & a-ketoglutaric acid & Succinic aicd & Fumaric acid \\
\hline WT & 621.9 & 0.058 & 1.8 & 9.1 & 10.2 & 0.081 \\
\hline cadA-21 & 667.8 & 0.059 & 1.6 & 8.0 & 11.0 & 0.074 \\
\hline$m f s A-2$ & 646.0 & 0.068 & 1.8 & 7.2 & 9.9 & 0.075 \\
\hline$m t-p f k A-5$ & 630.62 & 0.066 & 1.5 & 7.3 & 9.8 & 0.074 \\
\hline$m t t A-16$ & 590.8 & 0.067 & 1.8 & 7.6 & 15.2 & 0.094 \\
\hline citA-7 & 573.01 & 0.081 & 1.1 & 6.2 & 4.1 & 0.081 \\
\hline ATEG_01954-16 & 563.3 & 0.051 & 1.7 & 8.2 & 9.4 & 0.081 \\
\hline ATEG_09969-14 & 539.9 & 0.046 & 1.8 & 7.9 & 8.0 & 0.069 \\
\hline$a c o A-20$ & 535.85 & 0.055 & 1.2 & 6.6 & 7.9 & 0.070 \\
\hline gpdA-11 & 527.3 & 0.014 & 1.7 & 6.6 & 7.5 & 0.080 \\
\hline
\end{tabular}

${ }^{a}$ The citrate concentrations were estimated to be below $0.01 \mathrm{mM}$, while pyruvate, lactate and oxalate could not be detected in all samples.

Organic acids were separated on a Bio-Rad HPX-87H column by HPLC after 76-hr incubation, and detected by a refractive index detector and a UV-vis one (at $210 \mathrm{~nm}$ ). The standard curves of all organic acids were established using $0.5 \mathrm{mM}$ crotonic acid as an internal standard. 
other genes showed varied effects on itaconic acid production. Extracellular organic acids rather than itaconic acid were affected by overexpression of these genes to different extent. The genetic engineering approach will be useful for increasing itaconate production capacity and lowering the cost of industrial production.

\section{Methods}

\section{Materials}

Chemicals were obtained from Sigma, Merck or Ameresco. Oligonucleotides were synthesized by Shanghai Sangon Biotech Co. Ltd (China). Taq and pfu DNA polymerases, RevertAid Reverse Transcriptase, restriction endonucleases were purchased from Fermentas or New England BioLabs. The kits used for molecular cloning were bought from Omega Bio-tek Biotechnology. Difco ${ }^{\mathrm{Tm}}$ Potato Dextrose Agar was from BD. Trizol was from Invitrogen Life Technologies Corporation. DIG High Prime DNA Labelling and Detection Starter Kit I was purchased from RocheApplied Science. Hygromycin B was obtained from Solarbio Science Technology Co., Ltd (China).

\section{Plasmids, strains, media and cultivation conditions}

The plasmids pAN52-4 and pAN7-1 were kindly provided by Professor Punt from TNO Microbiology and Systems Biology (Netherlands) [24,42]. Spores were harvested from 7-day-old potato dextrose agar plate (PDA). Cultivation in shake flasks was carried out in $500 \mathrm{ml}$ shake flasks containing $55 \mathrm{ml}$ itaconic acid production medium (IPM) on a rotary shaker at $200 \mathrm{rpm}$ and $37^{\circ} \mathrm{C}[10]$.

\section{DNA manipulations and transformation}

General molecular biology techniques were carried out following the standard procedures [43]. The primers for the target genes from $A$. terreus were designed on the basis of the genome sequence of $A$. terreus $\mathrm{NIH} 2624$ (http://www.broadinstitute.org/annotation/genome/aspergillus_group/FeatureSearch.html). All primers were listed in Table S1 (Additional file 7).

The DNA fragments of $m t t A$ (ATEG_09970), cadA (ATEG_07991), ATEG_09969, gpdA (ATEG_09817), acoA (ATEG_03325) and citA (ATEG_07990) were amplified from the cDNA of $A$. terreus LYT10 using the respective primer pairs. The $m f s A$ gene was cloned as follows: The sequence of the cloned $m f s A$ fragment is slightly different from the annotated one at the 3' end when amplified using the primers $m f_{s} A-\mathrm{F}$ and $m f_{s} A-\mathrm{R} 1$. The primer $m f_{s} A-\mathrm{L}-\mathrm{R} 1$ was then designed at about 500 bp downstream of the annotated stop codon of $m f s A$, and the DNA fragment containing $m f s A$ was amplified from the genomic DNA of $A$. terreus LYT10. Based on the sequencing result, a series ofnew reverse primers ( $m f s A-\mathrm{L}-\mathrm{R} 2, m f s A-\mathrm{L}-\mathrm{R} 3, m f s A-\mathrm{L}-\mathrm{R} 4$, $m f s A-\mathrm{L}-\mathrm{R} 5)$ at the downstream of the annotated stop codon of $m f s A$ were redesigned to clone the $m f s A$ gene from the cDNA of $A$. terreus LYT10.The DNA fragment amplified using the primers $m f s A-\mathrm{F}$ and $m f s A-\mathrm{L}-\mathrm{R} 2$ covers the full ORF of the $m f_{s} A$ gene. The new introns and stop codon were identified, and the new sequence has been deposited with GenBank Accession No. KF305087. Finally, $m f s A$ was amplified using the primers $m f s A-\mathrm{F}$ and $m f s A-\mathrm{R} 2$ for further subcloning. The sequence identity between the cDNAs of $m f s A$ from $A$. terreus LYT10 and $A$. terreus $\mathrm{NIH} 2624$ is $80.5 \%$ (Additional file 8: Figure S7). The sequence identity between them is $81.5 \%$ at the protein level (Additional file 9: Figure S8). The difference is possibly due to annotation of the introns and stop codon for $m f s A$.

Truncated $p f k A$ in which 335 amino acids were removed at the C-terminus of PFK1 was amplified from the cDNA of $A$. niger Co827, and the phosphorylation site Thr 89 of $p f k A$ was mutated using the primers mt-pfk $A-\mathrm{F} / \mathrm{mt}-p f k A$ $\mathrm{R}$ as reported [23].

All DNA fragments were inserted at the restriction sites of NcoI and BamHI or HindIII of the vector pAN52-4 to obtain the pAN52-XX (XX, the different genes) plasmids respectively. The linearized plasmids by DraI and EcoRI were co-transformed with pAN7-1 into A. terreus LYT10 respectively [14]. Transformants were selected on the PDA-SH plates (PDA supplemented with 1.2 $\mathrm{M}$ sorbitol and $100 \mathrm{mg} / \mathrm{L}$ hygromycin B). The integrations of the target genes into the genome were confirmed by genomic PCR using the primers pAN-seq-F and pANseq-R. Five transformants for each gene were randomly selected for genomic PCR.

ATEG_01954 was amplified from the cDNA of $A$. terreus LYT10 and cloned into the vector $\mathrm{pG} 3 \mathrm{H}$ at the restriction sites of PciI and BglII [25]. The linearized plasmid was transformed into A. terreus LYT10. Transformants were selected on the PDA-SH plates. The integration of ATEG_01954 into the genome was confirmed by genomic PCR using the primers M13-47 and ATEG_01954-R1. Five transformants were randomly selected for genomic PCR.

The $h p h$ cassette was cut from the pSGF957 plasmid with $X b a \mathrm{I}$ and inserted into pAN52-cadA digested with $X b a I$ to get pAN52-cadA-hph. The linearized plasmids pAN52-cadA-hph and pAN52-mfs $A$ were co-transformed into A. terreus LYT10. The co-integrations of the cadA and $m f s A$ gene were confirmed by genomic PCR using the primers pAN-seq-F and cadA-R or mfsA-R.

Southern blot analysis was performed using DIG High Prime DNA Labelling and Detection Starter Kit I (Roche Applied Science) according to the manufacturer's instructions. Genomic DNAs of the selected cad $A$ - and $m f s A$-transformants (cadA-2, -5, -18, -21, -22, and $\left.m f_{s} A-2,-10,-17,-12,-24\right)$ were isolated by Fungal DNA Kit (Omega Bio-tek) and digested with $B a m H I$ at $37^{\circ} \mathrm{C}$ overnight. The DNA probes of $c a d A$ and 
$m f s A$ were amplified by $\mathrm{PCR}$ using the primer pairs CADt-F/CADt-R and MFSt-F/MFSt-R respectively.

\section{Analysis of the transformants}

The transformants, which can grow healthily on the PDA plates, were tested for itaconic acid production in $50 \mathrm{ml}$ IPM on a rotary shaker at $37^{\circ} \mathrm{C}$ for $76 \mathrm{~h}$. Each transformant was grown in three individual flaks, and the screening experiments for the important transformants were repeated once. The titers of itaconic acid produced were determined by HPLC (High Performance Liquid Chromatography) using an Aminex HPX-87-H column $(300 \mathrm{~mm} \times 7.8 \mathrm{~mm})$ detected at $210 \mathrm{~nm}$. The column was operated at $35^{\circ} \mathrm{C}$ with a mobile phase of $4 \mathrm{mM} \mathrm{H}_{2} \mathrm{SO}_{4}$ at a flow rate of $0.6 \mathrm{ml} / \mathrm{min}$. Authentic itaconic acid was used as a standard to calculate the final concentration of itaconic acid.

\section{Analysis of extracellular organic acids}

The concentrations of extracellular organic acids (itaconic, citric, cis-aconitic, pyruvic, malic, $\alpha$-ketoglutaric, lactic succinic, fumaric and oxalic acid) in the samples were determined by HPLC. Organic acids were separated on a Bio-Rad HPX-87H column by HPLC at $35^{\circ} \mathrm{C}$, using $5 \mathrm{mM}$ $\mathrm{H}_{2} \mathrm{SO}_{4}$ as the mobile phase at a flow rate of $0.6 \mathrm{~mL} \mathrm{~min}^{-1}$ and detected by a refractive index detector and a UV-vis one (at $210 \mathrm{~nm}$ ). The standard curves of all organic acids were established using $0.5 \mathrm{mM}$ crotonic acid as an internal standard.

\section{Itaconic acid production by cadA-21 at the demonstration scale}

The demonstration experiment by $c a d A-21$ was performed in IPM using a $35 \mathrm{~m}^{3}$ fermentor. A two-stage process including the vegetative phase and the production phase was used [40], and the vegetative phase was done for $16-18 \mathrm{hr}$ in $4 \mathrm{~m}^{3}$ fermentor. The only difference between the vegetative medium and IPM is glucose concentration: $40 \mathrm{~g} / \mathrm{L}$ versus $130 \mathrm{~g} / \mathrm{L}$. The fermentation stage was carried out with the following conditions: $37^{\circ} \mathrm{C}, 250 \mathrm{rev} / \mathrm{min}$, $1 \times 10^{7}$ spores $/ \mathrm{mL}$ (the initial spore concentration), and $20 \mathrm{~m}^{3} / \mathrm{min}$ (aeration). Samples were taken at regular intervals for analysis. Residual glucose was quantified using a biosensor (SBA-40C) from Biology Institute of Shandong Academy of Science (Jinan, China) following the standard protocol. The titer of produced itaconic acid was quantified by HPLC as above.

\section{Additional files}

Additional file 1: Figure S1. Genomic PCR analysis of the randomly selected transformants of each gene.

Additional file 2: Figure S2. Southern blot analysis of the cadA (A) - and $m f s A(B)$-transformants.
Additional file 3: Figure S3. Itaconic acid production by cadA (A) and mfsA (B) transformants. A. terreus LYT10 was as a reference (WT). The transformants were screened for itaconate production, and the itaonate titers after 76-hr incubation were determined by HPLC.

Additional file 4: Figure S4. Itaconic acid production by co-transformant of cadA and mfSA. A. terreus LYT10 was used as a reference (WT). The transformants were screened for itaconate production, and the itaonate titers after 76-hr incubation were determined by HPLC.

Additional file 5: Figure S5. HPLC analysis of itaconic acid produced by the best transformant cadA-21 (A, C) and A. terreus LYT10 (B, D). Samples were analyzed by HPLC after 76-hr incubation. A, C: the whole chromatograms; B, D: the enlarged ones for small peaks. Itaconic acid: the peak at $13.3 \mathrm{~min}$.

Additional file 6: Figure S6. Time courses of residual glucose (A) and itaconate production (B) for WT and strain cadA-21 at the pilot scale. The demonstration experiment was performed in a $35 \mathrm{~m}^{3}$ fermentor. A two-stage process including the vegetative phase and the production phase was applied, and the vegetative phase was done for 16-18 hr in $4 \mathrm{~m}^{3}$ fermentor. Samples were taken at regular intervals for analysis. In the inset, changes of glucose concentrations and itaconate titers during the final period were expanded.

Additional file 7: Table S1. The primers used in this study.

Additional file 8: Figure S7. Sequence alignment of $m f S A-D N A-L Y T 10$ (top), mfsA-cDNA-LYT10 (middle), and mfsA-cDNA-NIH2624 (bottom) LYT10, A. terreus LYT10; NIH2624, A. terreus NIH2624.

Additional file 9: Figure S8. Sequence alignment of MFS from $A$. terreus NIH 2624 (up) and A. terreus LYT10 (down) (at the protein level).

\section{Abbreviations}

ACO: Aconitase; CAD: cis-aconitate decarboxylase; CS: citrate synthase; IPM: itaconate production medium; MFS: Major Facilitator Superfamily; MTT: Mitochondrial tricarboxylic transporter.

\section{Competing interests}

The authors declare that they have no competing interests.

\section{Authors' contributions}

$\mathrm{XH}, \mathrm{XL}$ (XuefengLu), $\mathrm{YL}$, and JJL designed the experiments. $\mathrm{XH}$ and $\mathrm{XL}$ (Xia Li) performed the experiments. $X H, X L$ (Xuefeng $L u$ ), $Y L$, and JJL drafted the anuscript. All authors read and approved the final manuscript.

\section{Acknowledgements}

This project was supported by the Key Research Program of the Chinese Academy of Sciences (Grant No. KSZD-EW-Z-016).

\section{Author details}

${ }^{1}$ Key Laboratory of Biofuels, Shandong Provincial Key Laboratory of Energy Genetics, Qingdao Institute of Bioenergy and Bioprocess Technology, Chinese Academy of Sciences, No. 189 Songling Road, Qingdao 266101, China. ${ }^{2}$ Qingdao Kehai Biochemistry Co., Ltd, No. 198 Langyatai Road, Jiaonan, 266400 Qingdao, China. ${ }^{3}$ University of Chinese Academy of Sciences, Beijing 100049, China.

Received: 18 December 2013 Accepted: 5 August 2014 Published: 11 August 2014

\section{References}

1. Klement T, Buchs J: Itaconic acid A biotechnological process in change. Bioresour Technol 2013, 135:422-431.

2. Werpy T, Petersen G: Top Value Added Chemicals from Biomass - Volume I: Results of Screening for Potential Candidates from Sugars and Synthesis Gas. Oak Ridge, TN, USA: Department of Energy; 2004.

3. Lee JW, Kim HU, Choi S, Yi J, Lee SY: Microbial production of building block chemicals and polymers. Curr Opin Biotechnol 2011, 22:758-767.

4. Steiger MG, Blumhoff ML, Mattanovich D, Sauer M: Biochemistry of microbial itaconic acid production. Front Microbiol 2013, 4:23. 
5. Li A, van Luijk N, ter Beek M, Caspers M, Punt P, van der Werf M: A clone-based transcriptomics approach for the identification of genes relevant for itaconic acid production in Aspergillus. Fungal Genet Biol 2011, 48:602-611.

6. Bonnarme P, Gillet B, Sepulchre A, Role C, Beloeil J, Ducrocq C: Itaconate biosynthesis in Aspergillus terreus. J Bacteriol 1995, 177:3573-3578.

7. Jaklitsch WM, Kubicek CP, Scrutton MC: The subcellular organization of itaconate biosynthesis in Aspergillus terreus. J Gen Microbiol 1991, 137:533-539.

8. Kanamasa S, Dwiarti L, Okabe M, Park E: Cloning and functional characterization of the cis-aconitic acid decarboxylase (CAD) gene from Aspergillus terreus. Appl Microbiol Biotechnol 2008, 80:223-229.

9. Dwiarti L, Yamane K, Yamatani H, Kahar P, Okabe M: Purification and characterization of cis-aconitic acid decarboxylase from Aspergillus terreusTN484-M1. J Biosci Bioeng 2002, 94:29-33.

10. Kuenz A, Gallenmüller Y, Willke T, Vorlop KD: Microbial production of itaconic acid: developing a stable platform for high product concentrations. Appl Microbiol Biotechnol 2012, 96:1209-1216.

11. Carstensen F, Klement T, Büchs J, Melin T, Wessling M: Continuous production and recovery of itaconic acid in a membrane bioreactor. Bioresour Technol 2013, 137:179-187.

12. Okabe M, Lies D, Kanamasa S, Park EY: Biotechnological production of itaconic acid and its biosynthesis in Aspergillus terreus. Appl Microbiol Biotechnol 2009, 84:597-606.

13. Reddy CSK, Singh RP: Enhanced production of itaconic acid from corn starch and market refuse fruits by genetically manipulated Aspergillus terreus SKR10. Bioresour Technol 2002, 85:69-71.

14. Tevž G, Bencina M, Legisa M: Enhancing itaconic acid production by Aspergillus terreus. Appl Microbiol Biotechnol 2010, 87:1657-1664.

15. Lin YH, Li YF, Huang MC, Tsai YC: Intracellular expression of Vitreoscilla hemoglobin in Aspergillus terreus to alleviate effect of a short break in aeration duirng culture. Biotechnol Lett 2004, 26:1067-1072.

16. Li A, Pfelzer N, Zuijderwijk R, Brickwedde A, van Zeijl C, Punt P: Reduced by-product formation and modified oxygen availability improve itaconic acid production in Aspergillus niger. Appl Microbiol Biotechnol 2013, 97:3901-3911

17. van der Straat $L$, Tamayo-Ramos JA, Schonewille T, de Graaff LH: Overexpression of a modified 6-phosphofructo-1-kinase results in an increased itaconic acid productivity in Aspergillus niger. AMB Express 2013, 3:57.

18. van der Straat L, Vernooij M, Lammers M, van den Berg W, Schonewille T, Cordewener J, van der Meer I, Koops A, de Graaff LH: Expression of the Aspergillus terreus itaconic acid biosynthesis cluster in Aspergillus niger. Microb Cell Fact 2014, 13:11.

19. Li A, Pfelzer N, Zuijderwijk R, Punt P: Enhanced itaconic acid production in Aspergillus niger using genetic modification and medium optimization. BMC Biotechnol 2012, 12:57

20. Lubertozzi D, Keasling JD: Developing Aspergillus as a host for heterologous expression. Biotechnol Adv 2009, 27:53-75.

21. Meyer $V$ : Genetic engineering of filamentous fungi -Progress, obstacles and future trends. Biotechnol Adv 2008, 26:177-185.

22. Blumhoff ML, Steiger MG, Mattanovich D, Sauer M: Targeting enzymes to the right compartment: Metabolic engineering for itaconic acid production by Aspergillus niger. Metab Eng 2013, 19:26-32.

23. Capuder M, Šolar T, Benčina M, Legiša M: Highly active, citrate inhibition resistant form of Aspergillus niger 6-phosphofructo-1-kinase encoded by a modified pfkA gene. J Biotechnol 2009, 144:51-57.

24. Punt PJ, Zegers ND, Busscher M, Pouwels PH, van den Hondel CAMJJ: Intracellular and extracellular production of proteins in Aspergillus under the control of expression signals of the highly expressed Aspergillus nidulans gpdA gene. J Biotechnol 1991, 17:19-33.

25. Huang X, Lu X, Li J-J: Cloning, characterization and application of a native glyceraldehyde-3-phosphate dehydrogenase promoter for Aspergillus terreus. J Ind Microbiol Biotechnol 2014, 41:585-592.

26. Williamson D, Lu YJ, Gordon T, Sciot R, Kelsey A, Fisher C, Poremba C, Anderson J, Pritchard-Jones K, Shipley J: Relationship between MYCN copy number and expression in rhabdomyosarcomas and correlation with adverse prognosis in the alveolar subtype. J Clin Oncol 2005, 23:880-888.

27. Liao XG, Fang WG, Zhang YJ, Fan YH, Wu XW, Zhou Q, Pei Y: Characterization of a highly active promoter PBbgpd in Beauveria bassiana. Curr Microbiol 2008, 57:121-126.
28. Lubertozzi D, Keasling JD: Marker and promoter effects on heterologous expression in Aspergillus nidulans. Appl Microbiol Biotechnol 2006, 72:1014-1023.

29. Liao JC, Chang P-C: Genetically modified microorganisms for producing itaconic acid with high yields. In US2010/0285546 A1; 2010.

30. Wang J-H, Tsai S-I, Teng K: Producing itaconic acid in yeast using glycerol as the substrate. In US2011/0053232 A1; 2011.

31. Blumhoff M, Steiger MG, Marx H, Mattanovich D, Sauer M: Six novel constitutive promoters for metabolic engineering of Aspergillus niger. Appl Microbiol Biotechnol 2013, 97:259-267.

32. Picault N, Palmieri L, Pisano I, Hodges M, Palmieri F: Identification of a Novel Transporter for Dicarboxylates and Tricarboxylates in Plant Mitochondria. J Biol Chem 2002, 277:24204-24211.

33. Kaplan RS, Mayor JA, Gremse DA, Wood DO: High level expression and characterization of the mitochondrial citrate transport protein from the yeast Saccharomyces cerevisiae. J Biol Chem 1995, 270:4108-4114.

34. Campbell CD, Vederas JC: Biosynthesis of lovastatin and related metabolites formed by fungal iterative PKS enzymes. Biopolymers 2010, 93:755-763.

35. Lai LST, Hung CS, Lo CC: Effects of lactose and glucose on production of itaconic acid and lovastatin by Aspergillus terreusATCC 20542. J Biosci Bioeng 2007, 104:9-13.

36. Clomburg JM, Gonzalez R: Biofuel production in Escherichia coli: the role of metabolic engineering and synthetic biology. Appl Microbiol Biotechnol 2010, 86:419-434.

37. Guo ZP, Zhang L, Ding ZY, Wang ZX, Shi GY: Improving ethanol productivity by modification of glycolytic redox factor generation in glycerol-3-phosphate dehydrogenase mutants of an industrial ethanol yeast. J Ind Microbiol Biotechnol 2011, 38:935-943.

38. Holz M, Förster A, Mauersberger S, Barth G: Aconitase overexpression changes the product ratio of citric acid production by Yarrowia lipolytica. Appl Microbiol Biotechnol 2009, 81:1087-1096.

39. Ruijter GJ, Panneman H, Xu D, Visser J: Properties ofAspergillus niger citrate synthase and effects of CitA overexpression oncitric acid production. FEMS Microbiol Lett 2000, 184:35-40.

40. Riscaldati E, Moresi M, Federici F, Petruccioli M: Effect of $\mathrm{pH}$ and stirring rate on itaconate production by Aspergillu sterreus. J Biotechnol 2000, 83:219-230

41. Torres NV, Voit E, Gonzalez-Alcon C: Optimization of nonlinear biotechnological processes with linear programming: application to citric acid production by Aspergillus niger. Biotechnol Bioeng 1996, 49:247-258.

42. Punt PJ, Oliver RP, Dingemanse MA, Pouwels PH, van den Hondel CA: Transformation of Aspergillus based on the hygromycin B resistance marker from Escherichia coli. Gene 1987, 56:117-124.

43. Sambrook J, Fritsch EF, Maniatis T: Molecular Cloning A Laboratory Manual. 3rd edition. Cold Spring Harbor, NY: Cold Spring Harbor Press; 1989.

doi:10.1186/s12934-014-0119-y

Cite this article as: Huang et al.: Improving itaconic acid production through genetic engineering of an industrial Aspergillus terreus strain. Microbial Cell Factories 2014 13:119.

\section{Submit your next manuscript to BioMed Central and take full advantage of:}

- Convenient online submission

- Thorough peer review

- No space constraints or color figure charges

- Immediate publication on acceptance

- Inclusion in PubMed, CAS, Scopus and Google Scholar

- Research which is freely available for redistribution 